\title{
The role of the peritrophic matrix and red blood cell concentration in Plasmodium vivax infection of Anopheles aquasalis
}

\author{
Djane Clarys Baia-da-Silva ${ }^{1,2}$, Luis Carlos Salazar Alvarez ${ }^{1,2}$, Omaira Vera Lizcano ${ }^{3}$, Fabio Trindade Maranhão Costa ${ }^{4}$, \\ Stefanie Costa Pinto Lopes ${ }^{2,5}$, Alessandra Silva Orfanó ${ }^{6}$, Denner Oliveira Pascoal ${ }^{1}$, Rafael Nacif-Pimenta ${ }^{6}$, \\ Iria Cabral Rodriguez', Maria das Graças Vale Barbosa Guerra ${ }^{1,2}$, Marcus Vinicius Guimarães Lacerda²,5, \\ Nagila Francinete Costa Secundino ${ }^{6}$, Wuelton Marcelo Monteiro ${ }^{1,2}$ and Paulo Filemon Paolucci Pimenta ${ }^{1,2,6^{*}}$
}

\begin{abstract}
Background: Plasmodium vivax is predominant in the Amazon region, and enhanced knowledge of its development inside a natural vector, Anopheles aquasalis, is critical for future strategies aimed at blocking parasite development. The peritrophic matrix (PM), a chitinous layer produced by the mosquito midgut in response to blood ingestion, is a protective barrier against pathogens. Plasmodium can only complete its life-cycle, and consequently be transmitted to a new host, after successfully passing this barrier. Interestingly, fully engorged mosquitoes that had a complete blood meal form a thicker, well-developed PM than ones that feed in small amounts. The amount of red blood cells (RBC) in the blood meal directly influences the production of digestive enzymes and can protect parasites from being killed during the meal digestion. A specific study interrupting the development of the PM associated with the proteolytic activity inhibition, and distinct RBC concentrations, during the $P$. vivax infection of the New World malaria vector An. aquasalis is expected to clarify whether these factors affect the parasite development.

Results: Absence of PM in the vector caused a significant reduction in P. vivax infection. However, the association of chitinase with trypsin inhibitor restored infection rates to those of mosquitoes with a structured PM. Also, only the ingestion of trypsin inhibitor by non-chitinase treated mosquitoes increased the infection intensity. Moreover, the RBC concentration in the infected P. vivax blood meal directly influenced the infection rate and its intensity. A straight correlation was observed between RBC concentrations and infection intensity.

Conclusions: This study established that there is a balance between the PM role, RBC concentration and digestive enzyme activity influencing the establishment and development of $P$. vivax infection inside An. aquasalis. Our results indicate that the absence of PM in the midgut facilitates digestive enzyme dispersion throughout the blood meal, causing direct damage to $P$. vivax. On the other hand, high RBC concentrations support a better and thick, well-developed PM and protect $P$. vivax from being killed. Further studies of this complex system may provide insights into other details of the malaria vector response to $P$. vivax infection.
\end{abstract}

Keywords: Malaria, Plasmodium vivax, Peritrophic matrix, Trypsin, Chitinase, Hematocrit

\footnotetext{
* Correspondence: pimenta@cpqrr.fiocruz.br; pfppimenta@gmail.com

${ }^{1}$ Diretoria de Ensino e Pesquisa, Fundação de Medicina Tropical Dr. Heitor

Vieira Dourado, Manaus, AM, Brazil

${ }^{2}$ Programa de Pós-Graduação em Medicina Tropical, Universidade do Estado

do Amazonas, Manaus, AM, Brazil

Full list of author information is available at the end of the article
}

(c) The Author(s). 2018 Open Access This article is distributed under the terms of the Creative Commons Attribution 4.0 International License (http://creativecommons.org/licenses/by/4.0/), which permits unrestricted use, distribution, and reproduction in any medium, provided you give appropriate credit to the original author(s) and the source, provide a link to the Creative Commons license, and indicate if changes were made. The Creative Commons Public Domain Dedication waiver (http://creativecommons.org/publicdomain/zero/1.0/) applies to the data made available in this article, unless otherwise stated. 


\section{Background}

Malaria remains one of the most important public health problems worldwide. Overall, it is estimated that 3.2 billion people in 97 countries and territories are at risk of being infected with Plasmodium species and developing the disease [1]. Plasmodium falciparum is most prevalent in Africa and Plasmodium vivax is predominant in Southeast Asia and Latin America, mainly occurring in the Amazon region [1]. Approximately 70 Anopheles species are considered malaria vectors in the American continent [2]. Anopheles aquasalis is an important malaria vector that breeds in brackish marsh [3] and is distributed predominantly along the South and Central American areas where it is considered the primary coastal malaria vector of $P$. vivax [4-6]. Furthermore, $A n$. aquasalis is a species that has been colonized for several years [7] and in the last decade, it has been used as a good experimental model for interaction studies with $P$. vivax [8-13].

The peritrophic matrix (PM) is a semi-permeable and fibrous layer produced by secretion of intestinal epithelium of insects, including mosquitoes, in response to post-feeding midgut distension. The structured PM surrounds the food bolus inside the midgut and is considered responsible for several essential functions including preventing tissue damage, compartmentalization of ingested food, digestive enzyme flow control, and moreover as a protective barrier against pathogens [14]. Mosquitoes' PM is mainly composed of chitin, proteoglycans and proteins [15]. Chitin is mainly responsible for PM mechanical and tensile strengths and glycoproteins/proteoglycans are also involved in resistance, but mainly in PM permeability $[15,16]$. In haematophagous insects, distention due to blood meal ingestion stimulates midgut epithelial cells to generate a PM that remains until the end of the digestion process. In several species of mosquitoes of the genera Aedes, Culex and Anopheles, the PM starts to be synthetized between 3-6 h after blood ingestion and, in general, it is well structured around $24 \mathrm{~h}$, persisting until around $48 \mathrm{~h}$ when the digestion residues are excreted from the gut [17, 18]. Interestingly, fully engorged mosquitoes that had a complete blood meal form a thicker, well-developed PM than the ones that fed in small amounts $[19,20]$.

The ingestion of pathogenic microorganisms, such as viruses, bacteria and protozoa, may happen during the blood-feeding of a mosquito vector on infected individuals. These microorganisms then face the PM that acts as an important barrier in the midgut. Thus, ingested pathogens, including Plasmodium protozoa, can only complete their life-cycle and consequently be transmitted to a new vertebrate host after successfully passing the PM barrier, the first physical barrier in the vector midgut. Plasmodium gametocytes, after being ingested by the mosquito vector, complete their sexual cycle in the midgut lumen and develop into ookinetes, the mobile parasite form. The ookinetes then invade the PM, leaving behind the blood bolus, and get into the surrounding midgut epithelium. Plasmodium parasites start to invade the PM and reach the mosquito midgut epithelial cells around 18-24 h after the infective blood meal ingestion [21-23].

Ingested Plasmodium faces trypsin activities, one of the major digestive enzymes that process the blood meal inside the mosquito midgut, during the gametocyteookinete differentiation [24]. The amount of red blood cells $(\mathrm{RBC})$ in the blood meal directly influences the production of digestive enzymes $[21,22]$ and can protect parasites from being killed during the meal digestion $[23,25]$. However, it is well known that malaria causes anemia in infected people, likely affecting physical properties of the blood meal and further PM formation [26]. Furthermore, when digestive enzymes challenge ookinetes, they are also stimulated to synthetize a typical Plasmodium-specific chitinase to open a hole and cross the PM [27]. Experimental studies adding substances to the infective blood meal that interfere with PM synthesis or maintain a structured PM for a longer time have provided insights into the PM role during the Plasmodium life-cycle inside the vector [25]. These studies were developed with P. falciparum and murine or avian Plasmodium species [25, 28, 29].

The main aim of this work was to understand the roles of the PM and RBC concentrations in the infection of An. aquasalis with $P$. vivax. To our knowledge, there is no such information about similar studies developed with $P$. vivax in a natural mosquito vector. We sought to characterize the infection of the vector via infective blood meals containing chitinase, trypsin inhibitor and hematocrits at different concentrations. Detailed study interrupting the development of the PM associated to the inhibition of the proteolytic activity and the addition of distinct $\mathrm{RBC}$ concentrations during the infection of a natural vector by $P$. vivax was expected to clarify if these factors affect its vector competence or not. This study established that there is a balance between the PM role, $\mathrm{RBC}$ concentration and digestive enzyme activity influencing the establishment and development of $P$. vivax modulating the infection inside the New World malaria vector An. aquasalis.

\section{Methods}

\section{Anopheles aquasalis rearing and maintenance}

Mosquitoes were reared at the insectaries of the Laboratory of Medical Entomology at the Fundação de Medicina Tropical Dr Heitor Vieira Dourado (FMT-HVD), Manaus, and at the Instituto de Pesquisas René Rachou (Fiocruz-MG), Belo Horizonte, Brazil. Colonies were 
kept between $24-26{ }^{\circ} \mathrm{C}$ and $70-80 \%$ of relative humidity on a 12:12 h light-dark cycle. Larvae were hatched in room temperature water and ground TetraMin ${ }^{\circledR}$ fish food (Madison, Wisconsin, USA) was provided daily. Larvae were allowed to pupate and emerge into adults in an enclosed mesh-covered cage with water and fed $a d$ libitum in $10 \%$ sucrose solution until two days before the infective blood meals [11].

\section{Blood collection and ethics statement}

Adult volunteers (aged $>18$ years) residing in the region of Manaus, presenting at the FMT-HVD with microscopically confirmed $P$. vivax malaria, were invited to participate in the study. About $3 \mathrm{ml}$ of blood were collected by venipuncture and placed into a sterile heparinized vacutainer tube.

\section{Midgut dissection, histology and scanning electron microscopy (SEM)}

In order to observe the structure and the chitinase effect on the PM inside the midgut of An. aquasalis, two blood-feeding experiments were performed: (i) feeding with normal blood meal consisting of a blood sample of non-infected health donors (control group) and (ii) feeding with normal blood meal mixed with $1 \mathrm{U} / \mathrm{ml}$ Streptomyces chitinase (chitinase-treated group) as described elsewhere $[23,25,29,30]$. The blood meals were offered to groups of An. aquasalis for $30 \mathrm{~min}$ through membrane feeders, as described previously [11, 12]. Control and chitinase-treated mosquito groups were dissected and processed $24 \mathrm{~h}$ after the blood meal for histology or for SEM. Mosquitoes were immobilized at $4{ }^{\circ} \mathrm{C}$ and transferred to a Petri dish placed on ice. In order to conduct morphological observation, midguts were dissected in phosphate-buffered saline (PBS), $\mathrm{pH} 7.4$ under a stereomicroscope. These midguts were fixed at room temperature with $2.5 \%$ glutaraldehyde (GA) solution in $0.1 \mathrm{M}$ caccodylate buffer and after $24 \mathrm{~h}$ were routinely processed for histology or SEM. For histological observation, midguts were dehydrated and embedded in Historesin (Leica ${ }^{\circ}$, Wetzlar, Germany). 2- $\mu \mathrm{m}$ histological sections were obtained, stained with $1 \%$ toluidine blue solution for $3 \mathrm{~min}$ and mounted on glass slides to be analyzed and photographed under optical microscope [23, 30]. For SEM observation, the GA-fixed midguts were post-fixed with $1 \%$ osmium tetroxide solution containing $0.8 \%$ potassium ferricyanide for $2 \mathrm{~h}$. Next, they were dehydrated in serial crescent acetone solution and processed in a $\mathrm{CO}_{2}$ critical point device and assembled on stubs. The dried midguts were fractured with entomological stilettos and metallized with $20 \mathrm{~nm}$ gold particles to be observed and analyzed in a scanning electron microscope [31]. Histology and SEM were used to observe morphological aspects of the mosquito's PM.

\section{Oral $P$. vivax infection of An. aquasalis}

Adult mosquitoes were sugar starved overnight prior to infection via membrane-feeding assays. Briefly, $P$. vivax infected blood samples in diverse conditions, as described below, were offered to groups of 50 to $200 A n$. aquasalis for $30 \mathrm{~min}$ through membrane feeders at $37^{\circ}$ $\mathrm{C}$, as described previously $[11,12]$. Two sets of experiments were performed to study the PM role and the effect of RBC concentration in the mosquito infection by $P$. vivax.

To evaluate the PM role, blood meals of $P$. vivax-infected blood added with distinct substances were offered to the four different groups of An. aquasalis mosquitoes according to the addition of chitinase or soybean trypsin inhibitor: (i) Control (infected blood meal); (ii) Chitinase (infected blood samples plus $1 \mathrm{U} / \mathrm{ml}$ Streptomyces griseus chitinase); (iii) Trypsin inhibitor (infected blood samples plus $1 \mathrm{U} / \mathrm{ml}$ soybean trypsin inhibitor); and (iv) Chitinase + Trypsin inhibitor (infected blood samples plus chitinase and soybean trypsin inhibitor). All the infected blood samples that compose each experimental group were collected from the same patient.

Chitinase and trypsin inhibitor concentrations used were as previously described [23, 25, 31-33]. All reagents were from Sigma-Aldrich ${ }^{\circ}$ (Saint Louis, USA). Biological replicates were performed for each experimental condition.

To assess the effect of different RBC concentrations (distinct hematocrits) on An. aquasalis infection, patient blood samples were centrifuged (2000 $\mathrm{g}$ for $15 \mathrm{~min}$ ) and after removal of plasma, $P$. vivax-infected erythrocytes were enriched by Percoll gradient or magnetic column as previously described [34]. The enriched infected cells were divided equally in three parts and used to prepare $1 \mathrm{ml}$ of blood meal offered to three different groups of mosquitoes according to RBC concentrations: (i) $40 \%$ hematocrit: $400 \mu \mathrm{l}$ of non-infected RBC and $600 \mu \mathrm{l}$ of non-immune $\mathrm{AB}$ serum (control group); (ii) $30 \%$ hematocrit: $300 \mu \mathrm{l}$ of non-infected RBC and $700 \mu \mathrm{l}$ of non-immune $\mathrm{AB}$ serum; and (iii) 15\% hematocrit: $150 \mu \mathrm{l}$ of non-infected $\mathrm{RBC}$ and $850 \mu \mathrm{l}$ of non-immune $\mathrm{AB}$ serum. Therefore, the final gametocyte counts were the same for the 3 experimental groups. After the bloodfeeding experiments, only fully engorged mosquitoes were transferred to rearing containers. They were maintained in the insectary to be dissected at appropriated times after the blood meal for processing as described below. Biological replicates were performed for each experimental condition.

\section{Analyses of $P$. vivax infection of An. aquasalis}

Nine days after infective blood meals, midguts from all experimentally infected mosquito groups were dissected in PBS as described above, stained with $0.1 \%$ commercial Mercurochrome (Merbromin ${ }^{\circ}$, Saint Louis, USA), placed 
under a cover glass and examined for the presence of oocysts in an optical microscope. The infection rate (percentage of infected mosquito midguts) and the infection intensity (mean number of oocysts per midgut) on each mosquito were recorded and compared among the experimental groups.

\section{Statistical analysis}

Shapiro-Wilk test was used to verify the normality of the distribution. Multiple-sample comparisons were analyzed using one-way ANOVA. A nonparametric Kruskal-Wallis test with Dunn's multiple comparisons post-hoc test was used for intensity analysis, and for the infection rate with parametric distribution the ordinary one-way ANOVA with Turkey's multiple comparisons post-hoc test. Significant correlations with $P$ values $\leq 0.05$ were considered significant. All statistical analyses were performed using GraphPad Prism ${ }^{\circ}$ software (Prism 5.01; GraphPad Software Inc.).

\section{Results}

\section{Structure of An. aquasalis PM and the effect of the} exogenous chitinase

The An. aquasalis mosquito midgut $24 \mathrm{~h}$ after the normal blood meal (control group) revealed a well- developed PM as a coarse-thick and dense structure underneath the single midgut epithelium (Fig. 1a, c). At this time point, the PM is enclosed just above the midgut epithelium, segregating the entire blood meal. In contrast, An. aquasalis midguts $24 \mathrm{~h}$ after the chitinasecontaining blood meal showed a total absence of the PM, with the partially digested blood meal in direct contact with the midgut epithelium (Fig. 1b, d).

\section{PM role and digestive enzymes in $P$. vivax infection of $A n$. aquasalis}

The roles of chitinase and trypsin in P. vivax infection of An. aquasalis were analyzed by calculating the infection rate and intensity in different groups of treated/untreated mosquitoes. All experimental groups showed infected $P$. vivax mosquitoes but with distinct infection rates (ANOVA: $\left.F_{(3,8)}=10.63, P=0.0036\right)$ and infection intensities (Kruskal-Wallis H-test: $X^{2}=41.333, d f=3, P<$ 0.0001) (Fig. 2). Trypsin inhibitor group presented similar infection rate $(P=0.1672)$ and infection intensities $(P=$ $0.2059)$. The Chitinase group showed a significant reduction in infection rate $(P=0.0155)$ and infection intensity $(P<0.001)$ compared to Control group. On the other hand, the Chitinase + Trypsin group presented a similar
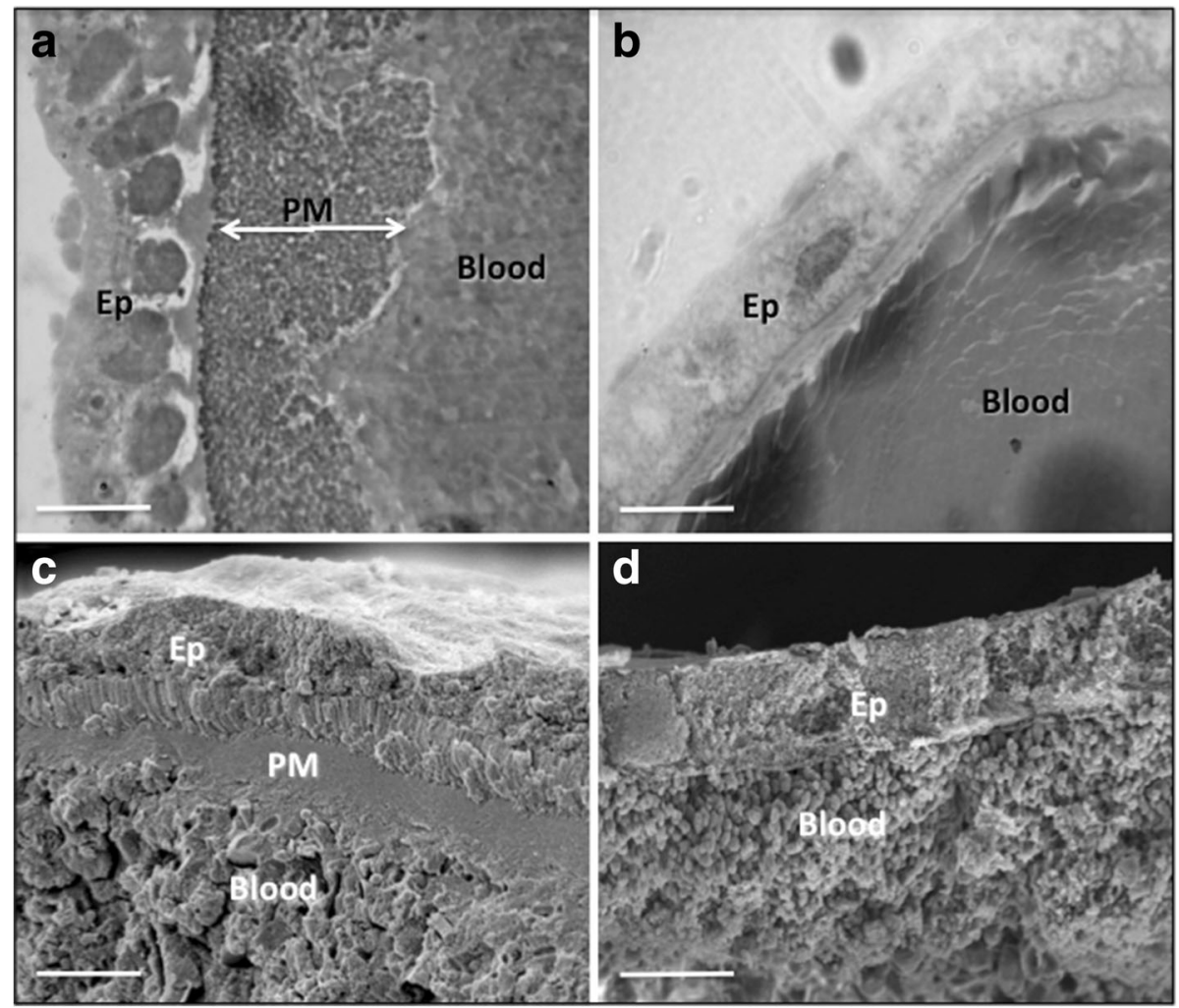

Fig. 1 Morphology of An. aquasalis midguts after normal and chitinase-containing blood meals. Histology (a) and SEM (c) of An. aquasalis midguts $24 \mathrm{~h}$ after a normal blood meal. The thick PM is visible isolating the midgut epithelium from the partially digested blood meals. Similar histology (b) and SEM (d) of An. aquasalis midguts $24 \mathrm{~h}$ after a chitinase-containing blood meal. The PM is absent inside the midgut, and the blood meal is in direct contact with the epithelium. Abbreviations: PM, peritrophic matrix; Ep, epithelium; Blood, blood meal. Scale-bars: a-d, $50 \mu \mathrm{m}$ 

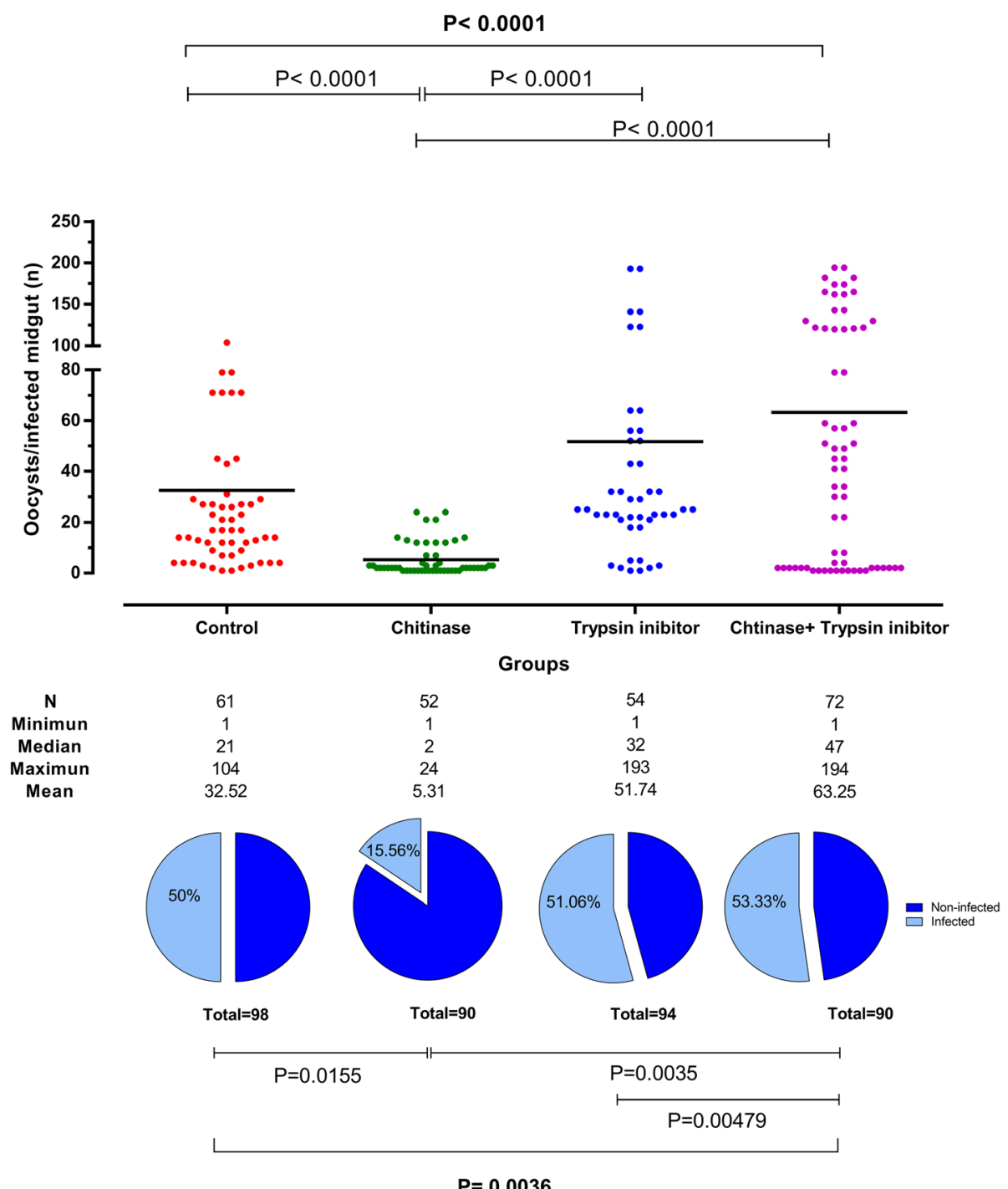

Fig. 2 Effect of exogenous chitinase and trypsin enzyme inhibitor on the infection of Anopheles aquasalis with Plasmodium vivax. The intensity of infection of each experimental group is presented in the top graph as the oocyst number per midgut (dots), the black lines represent the mean (Kruskal-Wallis H-test: $X^{2}=41.333, d f=3, P<0.0001$ ). The infection rate is represented in the bottom graph as the percentage of $P$. vivax infected mosquitoes (light blue pie section) (ANOVA: $F_{(3,8)}=10.63, P=0.0036$ )

infection rate $(P=0.660)$ and infection intensity $(P>$ $0.999)$ to those of Control group, and higher infection rate $(P=0.0035)$ and infection intensity $(P<0.001)$ when compared with the Chitinase group.

\section{Effect of RBC concentration in the P. vivax infection of $A$. aquasalis}

The role of RBC in P. vivax infection of An. aquasalis was analyzed by determination of the infection rate and intensity in groups of mosquitoes fed with the same amount of $P$. vivax gametocytes diluted in different concentration hematocrits (15\%, 30\% and 40\%). All experimental groups showed infected $P$. vivax mosquitoes but with distinct infection rates (Kruskal-Wallis H-test: $\chi^{2}=$
8.578, $d f=2, P=0.0093)$ and/or infection intensities (Kruskal-Wallis H-test: $\chi^{2}=19.090, d f=2, P<0.0001$ ) (Fig. 3). The $40 \%$ hematocrit (normal RBC) group of mosquitoes presented an infection rate of $48.9 \%$ and an infection intensity of 6.5 oocysts/infected midgut; whereas the $15 \%$ hematocrit (lowest RBC) group of mosquitoes showed a reduction in infection rate $(29.7 \%)$ and intensity of 4.2 oocysts/infected midgut $(P=0.0174$ and $P=0.011$, respectively). Moreover, the $30 \%$ hematocrit (intermediate $\mathrm{RBC}$ concentration) group presented a similar infection rate (44.9\%) and infection intensity of 7.4 oocysts/infected midgut to $40 \%$ hematocrit group ( $P$ $>0.999$ and $P=0.396)$ and higher infection intensity than the $15 \%$ hematocrit group $(P<0.001)$. 

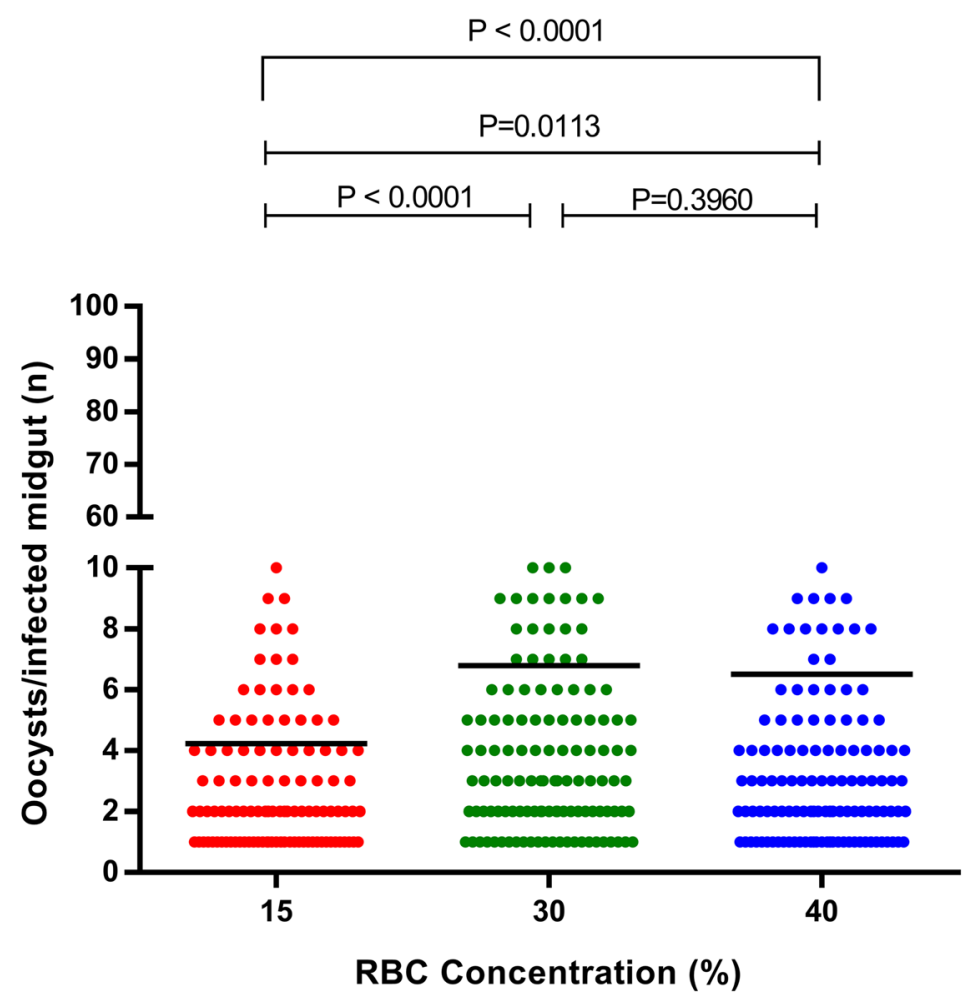

$\begin{array}{cccc}\mathbf{N} & 116 & 150 & 146 \\ \text { Minimun } & 1 & 1 & 1 \\ \text { Median } & 2 & 4 & 3 \\ \text { Maximun } & 39 & 100 & 52 \\ \text { Mean } & 4.23 & 7.41 & 6.52\end{array}$

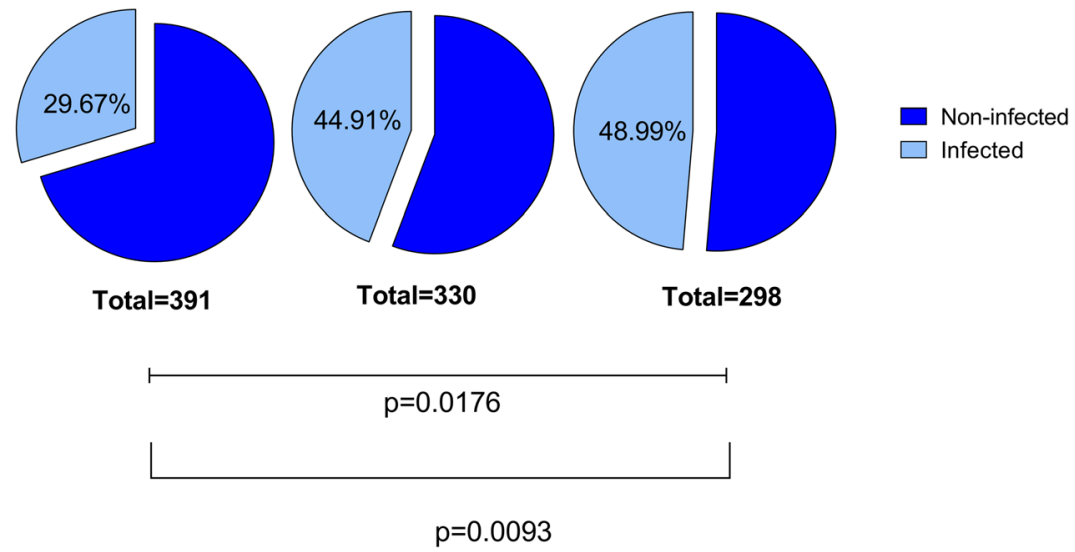

Fig. 3 Effect of blood meal hematocrit (RBC concentration) on the infection of An. aquasalis with P. vivax. The intensity of infection of each experimental group (red 15\% hematocrit, green 30\% hematocrit, blue $40 \%$ hematocrit) is presented in the top graph as the oocyst number per midgut (dots), the black lines represent the mean (Kruskal-Wallis H-test: $X^{2}=19.090, d f=2, P<0.0001$ ). The infection rate is represented in the bottom graph as the percentage of $P$. vivax infected mosquitos (light blue pie section) (Kruskal-Wallis H-test: $X^{2}=8.578, d f=2, P=0.0093$ )

\section{Discussion}

There is a significant knowledge gap about malarial infections by mosquito vectors from the American continent compared to vector-parasite pairs of African and Asian countries. Furthermore, most interaction studies were developed with the human malarial species $P$. falciparum or with avian and murine Plasmodium species. Only recently have interaction studies addressed interaction between Amazonian vectors and $P$. vivax, the most prevalent human parasite causing malaria in the 
New World. These studies have revealed important aspects of infection by Old World vectors [12].

The PM of mosquitoes acts as a physiological and physical barrier with several functions inside the midgut, including preventing tissue damage, controlling the digestive enzyme secretion and regulating pathogen infection as a vector response to either establishing or not a persistent infection. Some substances added to the ingested blood meal may disturb or even prevent PM formation. Since chitin is a main component of the PM structure, addition of exogenous chitinase has been demonstrated to prevent PM formation in blood-fed mosquitoes and other Diptera [25, 30-33].

Chitinase is a natural product synthesized by many organisms, especially fungi, which plays a key role in destabilization of chitinous structures, such as PM, or degradation of chitinous structures produced by the pathogen itself or its host [35]. For example, addition of chitinase from S. griseus to the blood meal offered to the sand fly Lutzomyia longipalpis [31] and to the mosquito Aedes aegypti [32] (vectors of Leishmania and important arboviruses, respectively) resulted in the absence of PM. Similarly, the histological and SEM observations presented here demonstrated that An. aquasalis mosquitoes that ingested S. griseus chitinase did not synthetize PM and, consequently, the mosquito midgut epithelium was in direct contact with the blood meal. Based on this initial knowledge, a series of experiments were developed in order to understand the role of the PM in P. vivax infection and the influence of the trypsin inhibitor on the American vector $A n$. aquasalis, including comparing its role in other malarial vector-parasite pairs.

Plasmodium species causative agents of murine and avian malaria have been used as models to understand the PM role in mosquito vectors. Development of $P$. berghei infection, a murine malarial parasite, in An. stephensi was unaffected by the absence of PM induced by chitinase ingestion [28]. However, preventing PM formation in Ae. aegypti by knocking down chitin synthase caused a lower infection rate by $P$. gallinaceum, an avian malarial parasite [29]. Moreover, increasing the thickness of the Ae. aegypti PM due to double blood-feedings only reduced the infection rate of $P$. gallinaceum [28]. This infection was completely blocked when ingestion of the chitinase inhibitor allosamidin resulted in a thicker PM [25]. Curiously, the same authors observed that the absence of PM resulting from ingestion of chitinase or polyoxin D did not affect $P$. gallinaceum infection [36]. These results suggest that in murine and avian malarial parasites the PM may act as a partial, but not absolute, barrier to parasite invasion of the mosquito midgut in distinct vector-Plasmodium pairs. Nevertheless, the present study demonstrated that the absence of the PM in the vector $A n$. aquasalis caused significant reduction in $P$. vivax infection. This fact emphasizes the importance of the PM role in the establishment of $P$. vivax infection in a relevant American vector.

One important PM role is controlling the flux of digestive enzymes produced by the mosquito midgut epithelium; it appears that PM limits the rate of digestion by reducing the diffusion of hydrolytic enzymes [37]. In Diptera, the porous PM induces a gradient of digestive enzyme activity in the blood meal inside the midgut, causing stronger digestive actions in the periphery than in the center of the digestive bolus [29, 31, 38-40]. The mosquito digestive tract contains a complex set of endoproteases and exopeptidases [41]; the major endoprotease acting in the blood meal digestion of mosquitoes is trypsin [42]. Proteolytic enzymes in mosquito midguts are located along the periphery of the blood bolus during the first $24 \mathrm{~h}$ after blood feeding [29]. Abraham \& Jacobs-Lorena [43] suggested that Plasmodium ookinetes in the outer parts of the blood meal, close to PM, die first due to the action of digestive enzymes, whereas ookinetes located closer to the interior of the blood meal, and consequently farther away from the effects of these enzymes, have a longer time to differentiate and survive enzymatic action. Interestingly, one of the first conclusive PM roles in protecting parasites has been reported in the sand fly vector Phlebotomus papatasi, infected with Leishmania major [31]. Although PM plays an important role in protecting the sand fly, it also creates a barrier that protects $L$. major from the action of digestive enzymes by limiting exposure of the parasite during first $24 \mathrm{~h}$ when it is deeply located in the center of the blood meal and vulnerable to proteolytic damage. In An. aquasalis, trypsin activity peaked between 12-24 $\mathrm{h}$ after blood-feeding, similar to other anophelines [43, 44], and in the same period when ingested $P$. vivax are transformed into motile forms, the ookinetes, in order to cross the PM and invade the midgut epithelium. Actually, gametes, zygotes and early non-differentiated ookinetes, the first Plasmodium forms inside the mosquito vectors in the first $24 \mathrm{~h}$ of infection, are very sensitive to digestive enzymes [22, 24]. The PM role is to provide a favorable midgut environment for Plasmodium survival and infection development. This study showed that the association of chitinase with trypsin inhibitor restored $P$. vivax infection rates to levels similar to that of mosquitoes that have a structured PM but with higher infection intensity, showing numerous oocysts in the mosquito midgut. Only the ingestion of trypsin inhibitor by non-chitinase treated mosquitoes resulted in a higher infection intensity. Indeed, these results suggest that the PM acts with enzyme digestive activities to regulate infection of $P$. vivax in An. aquasalis.

The RBC concentration in the blood meal ingested by hematophagous insects, including mosquitoes, affects 
the formation dynamics of the PM and, consequently, pathogen development in the vector [23, 45]. Anopheles stephensi and Ae. aegypti that are fully engorged with large blood meals in the midgut have a thicker, more well-developed PM than mosquitoes fed partially [46]. Also, there is a linear correlation between RBC concentration of the ingested infected blood meal and infectivity of Aedes aegypti by P. gallinaceum [45].

Anemia is a common feature in malaria infections [24]; moreover, a recent study revealed that moderate anemia increases the probability of vivax malaria patients to carry gametocytes [46]. In anophelines, the $\mathrm{RBC}$ component of the meal is concentrated by expelling plasma whilst feeding, a process termed prediuresis [47] that increases the number of infected cells ingested [48], thereby enhancing parasite transmission. Taylor \& Hurd [49] demonstrated using P. yoelii and An. stephensi that mosquitoes are unable to compensate for large decreases in RBC concentration by prediuresis, but a slight decrease in host hematocrit promoted a higher hemoglobin content in the vector, therefore a higher amount of erythrocytes uptake.

Our study demonstrated that very low RBC concentration diminishes $P$. vivax ability to establish infection in An. aquasalis. Additionally, we showed that a moderate decrease in hematocrit (30\%) did not alter $P$. vivax infectivity to the vector. It is important to highlight that the three mosquito groups fed with different RBC concentrations ingested the same amount of gametocytes per $\mathrm{ml}$ of blood meal. Nevertheless, the lower hematocrit (15\%) had a lower infection and intensity rates. Thus, RBC concentration modulates $P$. vivax infection. One possible explanation is the thicker, welldeveloped PM in An. aquasalis fed with high hematocrits, as described for Aedes aegypti infected with P. gallinaceum by [45]. Also, the dispersion and proximity of digestive enzymes have deleterious effects on the first Plasmodium forms, since the food bolus is small in the mosquitoes fed with low hematocrit.

\section{Conclusions}

An enhanced knowledge of $P$. vivax development inside the New World malaria vector An. aquasalis is critical for future strategies aimed at blocking parasite development. This study established that there is a balance between the PM role, RBC concentration and digestive enzyme activity, influencing the establishment and development of $P$. vivax inside An. aquasalis. One hypothesis is that the absence of PM, as well as a low RBC concentration, probably facilitate digestive enzyme dispersion throughout the blood meal inside the mosquito midgut causing direct damage to $P$. vivax. On the other hand, a high RBC concentration supports a thick welldeveloped PM and protects $P$. vivax from being killed by digestive enzymes inside the An. aquasalis midgut. Thus, here we provide new understanding of the mechanisms by which New World mosquito vector limit the development of $P$. vivax, which may lead to new methods for controlling malaria. Further studies of this complex system may provide insights into other details of the malaria vector response to infection.

\section{Abbreviations}

FMT-HVD: Fundação de Medicina Tropical Dr Heitor Vieira Dourado; GA: glutaraldehyde; PBS: phosphate-buffered saline; PM: peritrophic matrix; RBC: red blood cells; SEM: scanning electron microscopy

\section{Acknowledgements}

We acknowledge the collaboration of the local microscopists and the study subjects for their participation.

\section{Ethic approval and consent to participate}

This study was approved by the Committee of Ethics of the Fundação de Medicina Tropical Dr Heitor Vieira Dourado (CEP 686.297) and the Brazilian National Ethics Committee Board (3726). All participants were informed about the objectives of the study as well as potential risks and benefits of their participation. An informed consent form was signed by all study participants or by a parent or legal guardian in case of participants younger than 18 years. Children between 12 and 17 years signed an additional assent form. The procedures were approved by the FMT-HVD Ethics Review Board (Approval Number: 296.723/2014). After blood collection patients were treated according to Brazilian Health Ministry guidelines [50].

\section{Funding}

This study was partially funded by Bill and Melinda Gates Foundation (TransEpi Study), a grant from the National Institutes of Health (R01Al031478), and by the following Brazilian agencies: Foundation of the Institute Oswaldo Cruz (FIOCRUZ), Strategic Programme for Supporting Health Research (PAPES V), Brazilian Council for Scientific and Technological Development (CNPq), Minas Gerais State Research Support Foundation (FAPEMIG), São Psulo Research Foundation (FAPESP \# 2012/16525-2) and Amazonas State Research Support Foundation (PAPAC, Call 015/2014). The funders had no role in study design, data collection and analysis, decision to publish or preparation of the manuscript.

\section{Availability of data and materials}

All data generated from this study are included in this published article.

\section{Authors' contributions}

DCBS, ASO and RNP performed histology and scanning electron microscopy. LCSA and OVL performed gametocytes purification. DCBS, DOP and ICR were responsible for experimental infection and sample organization. DCBS, SCPL and WMM helped with statistical analysis. DCBS, MGBG, MVGL, SCPL, FTMC, NFCS, WMM and PFPP helped draft the manuscript and manuscript revision. DCBS, MVGL, NFCS, FTMC, SCPL, WMM and PFPP participated in study design, coordination, and writing the final version of the manuscript. All authors read and approved the final manuscript.

\section{Authors' information}

ASO, RNP, OVL and DCBS received PhD scholarships from one of the following Brazilian agencies: FAPEAM, FAPESP, FIOCRUZ, CNPq and CAPES. DCBS is a PhD student from the Graduation Program in Tropical Medicine from the State University of Amazonas, Brazil. PFPP, FTMC, NFCS and MVGL are senior fellows supported by CNPq.

Consent for publication

Not applicable.

Competing interests

The authors declare that they have no competing interests. 


\section{Publisher's Note}

Springer Nature remains neutral with regard to jurisdictional claims in published maps and institutional affiliations.

\begin{abstract}
Author details
'Diretoria de Ensino e Pesquisa, Fundação de Medicina Tropical Dr. Heitor Vieira Dourado, Manaus, AM, Brazil. 'Programa de Pós-Graduação em Medicina Tropical, Universidade do Estado do Amazonas, Manaus, AM, Brazil. ${ }^{3}$ Grupo de Investigación QUIBIO, Departamento de Biología, Universidad Santiago de Cali, Valle del Cauca, Colombia. ${ }^{4}$ Department of Genetics, Evolution and Bioagents, Institute of Biology, Universidade Estadual de Campinas, Campinas, SP, Brazil. ${ }^{5}$ Instituto Leônidas \& Maria Deane, Fiocruz, Manaus, AM, Brazil. 'Instituto de Pesquisas René Rachou, Fundação Oswaldo Cruz-Minas Gerais, Belo Horizonte, MG, Brazil.
\end{abstract}

Received: 3 November 2017 Accepted: 26 February 2018

Published online: 06 March 2018

\section{References}

1. WHO. World malaria report, vol. 2015. Geneva: World Health Organization; 2016.

2. Sinka ME, Bangs MJ, Manguin S, Rubio-Palis Y, Chareonviriyaphap T, Coetzee $M$, et al. A global map of dominant malaria vectors. Parasit Vectors. 2012;5:69.

3. Deane LM. Malaria vectors in Brazil. Mem Inst Oswaldo Cruz. 1986;81(Suppl. 2):5-14.

4. Berti J, Zimmerman R, Amarista J. Spatial and temporal distribution of anopheline larvae in two malarious areas in Sucre State, Venezuela. Mem Inst Oswaldo Cruz. 1993:88:353-62.

5. Chadee DD, Kitron U. Spatial and temporal patterns of imported malaria cases and local transmission in Trinidad. Am J Trop Med Hyg. 1999;61:513-7.

6. Laubach HE, Validum L, Bonilla JA, Agar A, Cummings R, Mitchell C, et al, Identification of Anopheles aquasalis as a possible vector of malaria in Guyana, South America. West Indian Med J. 2001;50:319-21.

7. Da Silva AN, Dos Santos CC, Lacerda RN, Santa Rosa EP, De Souza RT, Galiza D, et al. Laboratory colonization of Anopheles aquasalis (Diptera: Culicidae) in Belém, Pará, Brazil. J Med Entomol. 2006:43:107-9.

8. Bahia AC, Kubota MS, Tempone AJ, Pinheiro WD, Tadei WP, Secundino NF, et al. Anopheles aquasalis infected by Plasmodium vivax displays unique gene expression profiles when compared to other malaria vectors and plasmodia. PLoS One. 2010;5:e9795.

9. Bahia AC, Kubota MS, Tempone AJ, Araújo HR, Guedes BA, Orfano AS, et al. The JAK-STAT pathway controls Plasmodium vivax load in early stages of Anopheles aquasalis infection. PLoS Negl Trop Dis. 2011;5:e1317.

10. Bahia AC, Oliveira JH, Kubota MS, Araújo HR, Lima JB, Ríos-Velasquez CM, et al. The role of reactive oxygen species in Anopheles aquasalis response to Plasmodium vivax infection. PLoS One. 2013:8:e57014.

11. Ríos-Velasquez CM, Martins-Campos KM, Simões RC, Izzo T, dos Santos EV, Pessoa FAC, Lima JBP, et al. Experimental Plasmodium vivax infection of key Anopheles species from the Brazilian Amazon. Malar J. 2013;12:460.

12. Pimenta PF, Orfano AS, Bahia AC, Duarte AP, Ríos-Velasquez CM, Melo FF, et al. An overview of malaria transmission from the perspective of Amazon Anopheles vectors. Mem Inst Oswaldo Cruz. 2015;110:23-47.

13. Orfano AS, Nacif-Pimenta R, Duarte APM, Villegas LM, Rodrigues NB, Pinto $L C$, et al. Species-specific escape of Plasmodium sporozoites from oocysts of avian, rodent, and human malarial parasites. Malar J. 2016;15:394.

14. Hegedus D, Erlandson M, Gillott C, Toprak U. New insights into peritrophic matrix synthesis, architecture, and function. Annu Rev Entomol. 2009:54:285-302.

15. Toprak U, Baldwin D, Erlandson M, Gillott C, Hegedus DD. Insect intestinal mucins and serine proteases associated with the peritrophic matrix from feeding, starved and moulting Mamestra configurata larvae. Insect Mol Biol. 2010;19:163-75.

16. Tellam RL, Eisemann C. Chitin is only a minor component of the peritrophic matrix from larvae of Lucilia cuprina. Insect Biochem Mol Biol. 2000;30:1189-201.

17. Pascoa V, Oliveira PL, Dansa-Petretski M, Silva JR, Jacobs-Lorena M, Lemos FJA. Aedes aegypti peritrophic matrix and its interaction with heme during blood digestion. Insect Biochem Mol Biol. 2002;32:517-23.

18. Okuda K, de Souza Caroci A, Ribolla PE, de Bianchi AG, Bijovsky AT. Functional morphology of adult female Culex quinquefasciatus midgut during blood digestion. Tissue Cell. 2002;34:210-9.
19. Dinglasan RR, Devenport M, Florens L, Johnson JR, McHugh CA, DonnellyDoman M, et al. The Anopheles gambiae adult midgut peritrophic matrix proteome. Insect Biochem Mol Biol. 2009;39:125-34.

20. Freyvogel TA, Staubli $W$. The formation of the peritrophic membrane in Culicidae. Acta Trop. 1965;22:118-47.

21. Noriega FG, Edgar KA, Googman WG, Shah DK, Wells MA. Neuroendocrine factors affecting the steady-state levels of early tripsin mRNA in Aedes aegypti. J Insect Physiol. 2001;47:515-22.

22. Noriega FG, Wells MA. A molecular view of trypsin synthesis in the midgut of Aedes aegypti. J Insect Physiol. 1999;45:613-20.

23. Pimenta PF, Modi GB, Pereira ST, Shahabuddin M, Sacks DL. A novel role for the peritrophic matrix in protection Leishmania from the hydrolytic activities of the sand fly midgut. Parasitology. 1997;115:359-69.

24. Baton LA, Ranford-Cartwright LC. Ookinete destruction within the mosquito midgut lumen explains Anopheles albimanus refractoriness to Plasmodium falciparum (3D7A) oocyst infection. Int J Parasitol. 2012;42:249-58.

25. Shahabuddin M, Toyoshima T, Aikawa M, Kaslow DC. Transmission-blocking activity of a chitinase inhibitor and activation of malarial parasite chitinase by mosquito protease. Proc Natl Acad Sci USA. 1993:90:4266-70.

26. Haldar K, Malaria MN. erythrocytic infection, and anemia. Hematology Am Soc Hematol Educ Program. 2009:87-93.

27. Huber M, Cabib E, Miller LH. Malaria parasite chitinase and penetration of the mosquito peritrophic membrane. Proc Natl Acad Sci USA. 1991;88:2807-10.

28. Billingsley PF, Rudin W. The role of the mosquito peritrophic membrane in bloodmeal digestion and infectivity of Plasmodium species. J Parasitol. 1992; 78:430-40.

29. Kato N, Mueller CR, Fuchs JF, Mcelroy K, Wessely V, Higgs S, et al. Evaluation of the function of a type I peritrophic matrix as a physical barrier for midgut epithelium invasion by mosquito-borne pathogens in Aedes aegypti. Vector Borne Zoonotic Dis. 2008:8:701-12.

30. Secundino NF, Eger-Mangrich I, Braga EM, Santoro MM, Pimenta PF. Lutzomyia longipalpis peritrophic matrix: formation, structure, and chemical composition. J Med Entomol. 2005;42:928-38.

31. Pimenta PF, De Souza W. Leishmania mexicana amazonensis: Surface charge of amastigote and promastigote forms. Exp Parasitol. 1983;56:194-206.

32. Filho BP, Lemos FJ, Secundino NF, Páscoa V, Pereira ST, Pimenta PF. Presence of chitinase and beta- $\mathrm{N}$-acetylglucosaminidase in the Aedes aegypti. a chitinolytic system involving peritrophic matrix formation and degradation. Insect Biochem Mol Biol. 2002;32:1723-9.

33. Weiss BL, Savage AF, Griffith BC, Wu Y, Aksoy S. The peritrophic matrix mediates differential infection outcomes in the tsetse fly gut following challenge with commensal, pathogenic, and parasitic microbes. J Immunol. 2014:193:773-82.

34. Vera O, Brelas de Brito P, Albrecht L, Martins-Campos KM, Pimenta PF, Monteiro WM, et al. Purification methodology for viable and infective Plasmodium vivax gametocytes that is compatible with transmissionblocking assays. Antimicrob Agents Chemother. 2015:59:6638-41.

35. Bhattacharya D, Nagpure A, Gupta RK. Bacterial chitinases: properties and potential. Crit Rev Biotechnol. 2007:27:21-8.

36. Shahabuddin M, Kaidoh T, Aikawa M, Kaslow DC. Plasmodium gallinaceum mosquito peritrophic matrix and the parasite - vector compatibility. Exp Parasitol. 1995;81:386-93.

37. Villalon JM, Ghosh A, Jacobs-Lorena M. The peritrophic matrix limits the rate of digestion in adult Anopheles stephensi and Aedes aegypti mosquitoes. J Insect Physiol. 2003;49:891-5.

38. Terra WR, Ferreira C, Baker JE. Compartmentalization of digestion. In: Lehane MJ, Billingsley PF, editors. Biology of the insect midgut. Dordrecht: Springer; 1996. p. 206-35

39. Terra WR, Ferreira C. Insect digestive enzymes: properties, compartmentalization and function. Comp Biochem Physiol. 1994;109:1-62.

40. Edwards M, Jacobs-Lorena M. Permeability and disruption of the peritrophic matrix and caecal membrane from Aedes aegypti and Anopheles gambiae mosquito larvae. J Insect Physiol. 2000;46:1313-20.

41. Clements AN. The biology of mosquitoes. Vol. 1 Development, nutrition and reproduction. UK: Chapman \& Hall; 1992.

42. Graf $\mathrm{R}$, Briegel $H$. Comparison between aminopeptidase and trypsin activity in blood fed females of Aedes aegypti. Rev Suisse Zool. 1982;89:845-50.

43. Abraham EG, Jacobs-Lorena M. Mosquito midgut barriers to malaria parasite development. Insect Biochem Mol Biol. 2004;34:667-71.

44. Chadee DD, Beier JC. Blood-engorgement kinetics of four anopheline mosquitoes from Trinidad, West Indies. Ann Trop Med Parasitol. 1995;89:55-62. 
45. Rosenberg R, Koontz LC, Alston K, Friedman FK. Plasmodium gallinaceum: erythrocyte factor essential for zygote infection of Aedes aegypti. Exp Parasitol. 1984;57:158-64.

46. Koepfli C, Robinson $\perp$, Rarau P, Salib M, Sambale N, Wampfler R, et al. Blood-stage parasitaemia and age determine Plasmodium falciparum and $P$. vivax gametocytaemia in Papua New Guinea. PLoS One. 2015;10:e0126747.

47. Briegel H, Rezzonico L. Concentration of host blood protein during feeding by anopheline mosquitoes (Diptera: Culicidae). J Med Entomol. 1985;22:612-8.

48. Vaughan JA, Noden BH, Beier JC. Concentration of human erythrocytes by anopheline mosquitoes (Diptera: Culicidae) during feeding. J Med Entomol. 1991;28(6):780

49. Taylor PJ, Hurd $\mathrm{H}$. The influence of host haematocrit on the blood feeding success of Anopheles stephensi: implications for enhanced malaria transmission. Parasitology. 2001;122:491-6.

50. Ministério da Saúde. Secretaria de Vigilância em Saúde. Departamento de Vigilância Epidemiológica. Guia prático de tratamento da malária no Brasil/ Ministério da Saúde, Secretaria de Vigilância em Saúde, Departamento de Vigilância Epidemiológica. Braślia: Ministério da Saúde; 2010.

\section{Submit your next manuscript to BioMed Central} and we will help you at every step:

- We accept pre-submission inquiries

- Our selector tool helps you to find the most relevant journal

- We provide round the clock customer support

- Convenient online submission

- Thorough peer review

- Inclusion in PubMed and all major indexing services

- Maximum visibility for your research

Submit your manuscript at www.biomedcentral.com/submit 